\title{
Italiano in autostrada
}

\section{Vittorio Coletti}

PUBBLICATO: 31 DICEMBRE 2019

\section{Ottobre 2019}
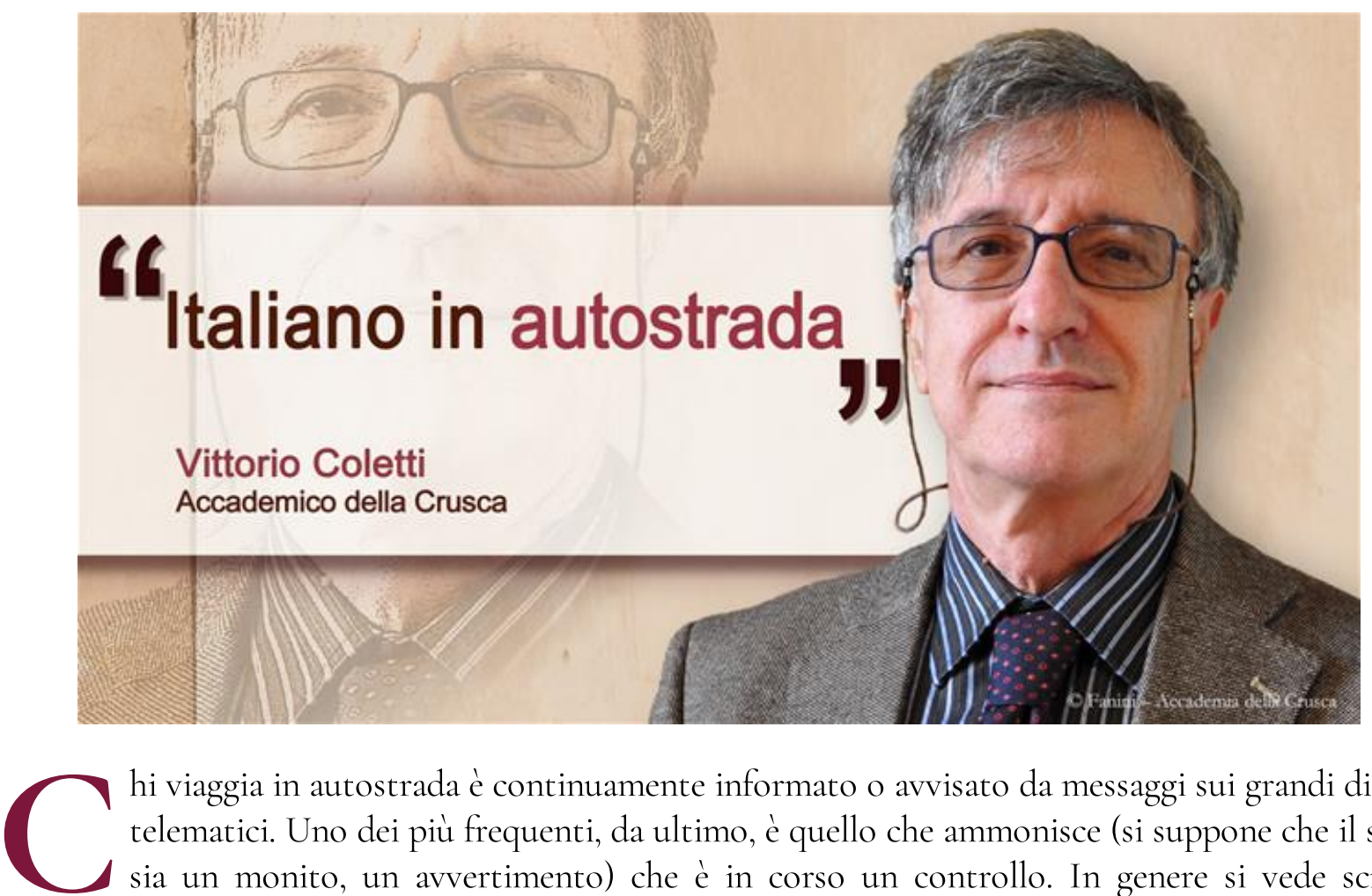

hi viaggia in autostrada è continuamente informato o avvisato da messaggi sui grandi display telematici. Uno dei più frequenti, da ultimo, è quello che ammonisce (si suppone che il senso sia un monito, un avvertimento) che è in corso un controllo. In genere si vede scritto: "Controllo elusione (pagamento) pedaggio" o anche "Controllo mancato (pagamento) pedaggio" o anche "Accertamenti mancato pedaggio". Evidentemente i comunicatori dei concessionari delle autostrade non sanno che non si controlla l'assenza di qualcosa, ma la sua presenza. Il controllo accerta l'osservanza di norme; la sanzione della loro inosservanza ne è la conseguenza. Il controllore verifica il possesso del biglietto e punisce la sua mancanza, non il contrario! A rigore, il "controllo o accertamento (del) mancato (pagamento del) pedaggio" dovrebbe significare che si verifica se davvero non lo si è pagato e comportare che si sanziona chi lo ha fatto! Se si vuole parlare della mancanza o dell'elusione del pedaggio si dovrebbe scrivere: "Controlli o Accertamenti antielusione (del) pedaggio". Ma è molto più semplice: "Controlli pagamento (del) pedaggio".

È solo un'imprecisione l'avviso che ci si sta per avviare, nel senso di marcia che si percorre, verso un tratto di autostrada ridotta a una sola corsia, essendo chiusa l'altra per lavori o altro: "Carreggiata unica". In realtà, poiché la carreggiata è tutta la strada (a due o tre corsie) in una data direzione di marcia, unica è la corsia cui ci si immette. La carreggiata è ridotta. Ma in autostrada si confonde spesso la carreggiata, che è la sezione del tracciato che va in una delle due direzioni, con la corsia, che è una parte della carreggiata. È vero che carreggiata può stare anche per corsia; essere cioè, come scrive il GRADIT: "ciascuno dei due settori corrispondenti a opposti sensi di marcia in cui può essere divisa (una strada)", ma questo vale per le vie a doppio senso di marcia, cioè appunto a carreggiata unica. In autostrada, quasi ovunque a tracciati separati a seconda delle due direzioni, carreggiata indica quello di uno dei due sensi di marcia ed è perlopiù individuata con i punti cardinali (carreggiata sud, ovest ecc.). Quando lavori o altro riducono una carreggiata a due corsie, eliminandone una, la corsia 
che resta è l'unica. Diverso il caso in cui su una sola carreggiata si procede nei due sensi opposti, uno per corsia: in questo caso è corretto "carreggiata unica"; ma va forse meglio "carreggiata a doppio senso di marcia".

Più grave l'imprecisione frequentissima dell'avviso di "Scambio di carreggiata", quando si sta per essere deviati su una corsia della carreggiata opposta. In tal caso non si tratta di scambio, ma di cambio. Se fosse uno scambio noi ci prenderemmo la carreggiata di chi va in senso contrario al nostro e daremmo loro la nostra! Scambio è un cambio reciproco. È vero che nel lessico ferroviario scambio è il nome del congegno che regola la semplice deviazione da un binario all'altro. Ma in ferrovia è un tecnicismo in uso da fine Ottocento e, per di più, c’è reciprocità tra i due binari e sensi di marcia, che sono fissati in maniera preferenziale (i treni procedono in Italia, di norma, all'inglese, tenendo la sinistra) ma non immodificabile (le linee sono spesso oggi "banalizzate", cioè i treni possono percorrere indifferentemente uno stesso binario, a prescindere dal senso di marcia); mentre in autostrada no, o solo eccezionalmente. Quellas, dunque, è decisamente di troppo. In autostrada è bene rispettare, oltre i limiti di velocità, anche l'italiano.

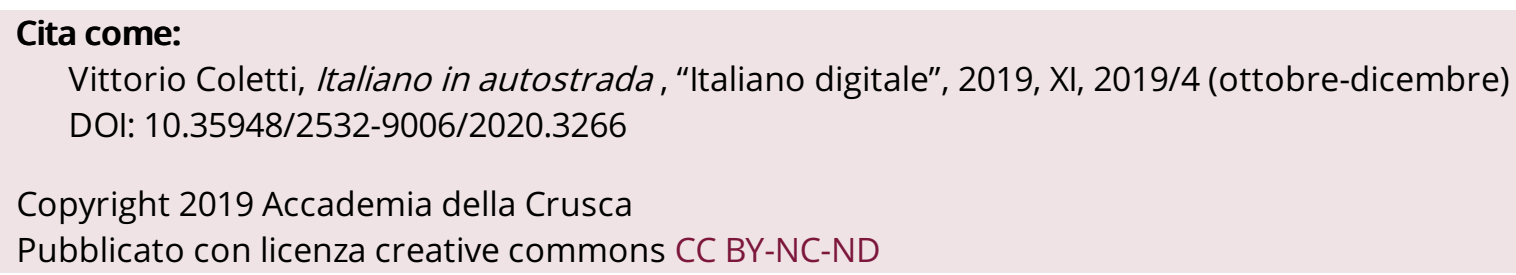

